\title{
Capacidade antioxidante em resíduos da indústria cafeeira
}

\author{
Antioxidant capacity in coffee industry residues
}

\section{Autores | Authors}

*Lady Rossana PALOMINO

GARCÍA

Universidade Estadual Paulista (UNESP)

Programa de Pós-graduação em

Engenharia e Ciência de Alimentos

Rua Cristóvão Colombo, 2265, Jardim Nazareth CEP: 15054-000

São José do Rio Preto/SP - Brasil e-mail: ladypalominog@gmail.com

Vanildo Luiz DEL BIANCHI

Universidade Estadual Paulista (UNESP) Departamento de Engenharia e Tecnologia de Alimentos

São José do Rio Preto/SP - Brasil e-mail:vanildo@ibilce.unesp.br

*Autor Correspondente / Corresponding Author

Recebido: Jun. 30, 2015

Aprovado: Dez. 07, 2015

\section{Resumo}

Este trabalho teve como objetivo a recuperação de compostos fenólicos a partir de resíduos da indústria cafeeira e a avaliação de sua capacidade antioxidante. Os resíduos (casca, polpa e borra de café) foram obtidos de diferentes etapas do processamento industrial do café. Os extratos fenólicos foram obtidos usando-se uma mistura de acetona e água. O teor de compostos fenólicos variou entre 72,88 e 159,50 $\mathrm{mg} \mathrm{AG} \mathrm{g}^{-1}$ resíduo. Mediante cromatografia líquida de alta eficiência foi detectado e quantificado o ácido clorogênico na casca e na polpa de café. Foi avaliada a capacidade antioxidante dos extratos fenólicos, verificando-se sua capacidade sequestrante de radicais DPPH e sua ação redutora sobre radicais peroxila. O resíduo com maior capacidade antioxidante foi a casca de café. Esses resultados sugerem a possibilidade de reutilização dos resíduos da indústria do café para a obtenção de compostos antioxidantes, pois tais compostos têm inúmeras aplicações nas áreas de alimentos, cosméticos e farmacêutica.

Palavras-chave: Resíduos de café; Compostos fenólicos; Ácido clorogênico; Radical livre DPPH; Radical peroxila.

\section{Summary}

The aim of this study was to recover the phenolic compounds from coffee industry residues and evaluate their antioxidant capacity. The residues (husk, pulp and spent coffee) were obtained from different steps of the industrial processing of coffee. The phenolic extracts were obtained using a mixture of acetone and water and the phenolic compound contents ranged between 72.88 and $159.50 \mathrm{mg}$ GA $\mathrm{g}^{-1}$ residue. Chlorogenic acid was detected and quantified in the coffee husk and pulp by high performance liquid chromatography (HPLC). The antioxidant capacity of the phenolic extracts was evaluated and they showed DPPH radical scavenging ability and reducing action against peroxyl radicals. The residue with the highest antioxidant capacity was the coffee husk. The results suggest the possibility of reusing the coffee industry residues to obtain antioxidant compounds, since these compounds have numerous applications in the food, cosmetic and pharmaceutical industries.

Key words: Coffee residues; Phenolic compounds; Chlorogenic acid; DPPH free radical; Peroxyl radical. 


\section{Introdução}

A exposição direta dos resíduos gerados pela agroindústria ao meio ambiente representa uma das causas principais de poluição ambiental e também significa uma perda de biomassa, que poderia ser utilizada para a produção ou recuperação de diferentes metabólitos com valor comercial. Recentemente tem-se incrementado o interesse na utilização eficiente dos resíduos agroindustriais, assim como na agregação de valor a eles (PANDEY et al., 2000; AJILA et al., 2011). De fato, esses resíduos podem ser uma fonte alternativa de antioxidantes naturais, que são considerados seguros quando comparados com os antioxidantes sintéticos (MURTHY; NAIDU, 2012).

A indústria cafeeira gera diferentes resíduos, dependendo do tipo de processamento e da etapa do beneficiamento do grão. Existem dois métodos de processamento industrial da cereja de café: a via seca e a via úmida. Dependendo do método utilizado, seco ou úmido, os principais resíduos recebem o nome de casca e polpa, respectivamente (FAN et al., 2000; MURTHY; NAIUDU, 2012). Mas as operações, que abrangem até a produção de café solúvel, envolvem vários tipos de resíduos além dos mencionados, como o pergaminho, o tegumento ou película e a borra de café.

Estudos têm demonstrado a presença de compostos fenólicos na casca e na polpa de café. Clifford e Ramirez-Martinez (1991) reportaram a presença de ácidos clorogênicos, cafeína e ácido protocatecuico na polpa de café Arabica, Robusta e de dois de seus híbridos. Ramirez-Coronel et al. (2004) encontraram quatro classes principais de polifenóis na polpa de café Arábica: flavan 3-ois, ácidos hidroxicinâmicos, flavonois e antocianidinas. A casca de café Arábica foi estudada como fonte de antocianinas por Prata e Oliveira (2007).

A borra de café é um resíduo com tamanho de partícula muito fino e altas umidade, carga orgânica e acidez, gerada durante a produção de café instantâneo. Cerca de $50 \%$ da produção de café no mundo é usada para o preparo de café solúvel, portanto, a borra de café é gerada em grandes proporções, com uma produção anual de 6 milhões de toneladas. Um dos principais problemas encontrados pela indústria de café é a disposição desses resíduos, que até o momento inclui descarga no esgoto ou nos aterros sanitários, incineração, alimentação do gado e utilização como enchimento e adsorvente em material termofixo (RAMALAKSHMI et al., 2009; MUSSATTO et al., 2011). Esse resíduo contém cafeína, taninos e polifenóis, embora em menores quantidades que a casca e a polpa (FAN et al., 2000). Atividades biológicas têm sido avaliadas nos extratos de borra de café, os quais têm mostrado expressiva atividade antirradicalar, antioxidante e antitumoral, embora apenas uma limitada ação anti-inflamatória e antialérgica (RAMALAKSHMI et al.,
2009). Suas propriedades antioxidantes poderiam ser consequência da presença de cafeína, trigonelina e ácidos clorogênicos (ESQUIVEL; JIMÉNEZ, 2012).

Murthy e Naidu (2012) analisaram a polpa, a casca, o tegumento e a borra de duas variedades de café (Arábica e Robusta), concluindo que esses resíduos contêm quantidades apreciáveis de compostos bioativos com capacidade antioxidante e estabelecendo, mediante análises de cromatografia líquida de alta eficiência (CLAE), que o principal componente é o ácido clorogênico, presente em teores na faixa de 10-23\%.

Visando estabelecer diferenças entre os resíduos gerados nos dois processos do beneficiamento do grão de café e também com o resíduo final do processamento de café solúvel, foi avaliado o teor de compostos fenólicos nos extratos obtidos a partir desses resíduos e estudadas suas propriedades antioxidantes.

\section{Material e métodos}

\subsection{Materiais}

Os resíduos de café utilizados nesta pesquisa foram obtidos de diferentes processamentos da cereja e do grão de café da variedade Robusta. A casca de café, que provém do processo seco da cereja, e a borra de café, oriunda do processamento do grão para a obtenção do café solúvel, foram cedidas pela indústria Cocam, Companhia de Café Solúvel e Derivados (Catanduva, São Paulo, Brasil). A polpa de café, gerada na etapa de despolpagem do processo úmido, foi adquirida em uma fazenda cafeeira na Colômbia (Timbío, Cauca). Os padrões: ácido clorogênico (ACG), rutina, quercetina, ácido gálico $(A G)$ e os reagentes: 2,2-difenil-1-picril-hidrazil (DPPH), dicloridrato de 2, 2'-azobis(2-metilpropanimidamida) (AAPH) 97\%, sal trissódico do ácido-8-hidroxipireno-1,3,6-trisulfônico (piranina) foram fornecidos pela Sigma (St. Louis, MO, EUA). O reagente Folin-Ciocalteu grau analítico foi fornecido pela Dinâmica (Diadema, SP, Brasil). A acetona grau analítico foi adquirida da Synth (Diadema, SP, Brasil). Os solventes grau HPLC foram fornecidos pela J. T. Baker (Phillipsburg, NJ, USA) e foi usada água deionizada purificada em sistema Milli-Q (Millipore, Bedford, MA, USA).

A solução do tampão fosfato salino (PBS) a pH 7,4 oi preparada com a adição de $8 \mathrm{~g}$ de $\mathrm{NaCl}, 0,2 \mathrm{~g} \mathrm{KCl}, 1,44 \mathrm{~g}$ de $\mathrm{Na}_{2} \mathrm{HPO}_{4}$ e 0,24 $\mathrm{g} \mathrm{KH}_{2} \mathrm{PO}_{4}$ em $1 \mathrm{~L}$ de água.

\subsection{Obtenção dos extratos acetônicos}

Foi utilizada a metodologia de extração previamente estabelecida por Palomino e Del Bianchi (2015). Antes de serem utilizados, os resíduos foram secos em estufa a $60^{\circ} \mathrm{C}$ até obter-se peso constante, sendo posteriormente triturados. 
Pesaram-se $10 \mathrm{~g}$ de cada resíduo, adicionando-se $200 \mathrm{~mL}$ de acetona-água (80:20 v/v). As amostras foram colocadas em frascos de Erlenmeyer em uma incubadora Shaker (Lucadema, modelo LUCA-222) a $40^{\circ} \mathrm{C}$ e $120 \mathrm{rpm}$ por $30 \mathrm{~min}$. O sobrenadante foi filtrado e o material sólido submetido a uma segunda extração com $200 \mathrm{~mL}$ de solvente. A seguir juntaram-se os sobrenadantes, centrifugando-os por 20 min a $5.000 \mathrm{rpm}$ e $0{ }^{\circ} \mathrm{C}$ em uma centrífuga (Hettich ${ }^{\circledast}$, modelo Rotina 420R), filtrando-se em seguida. Uma parte do sobrenadante foi reservada para a determinação dos compostos fenólicos totais e a outra, concentrada em evaporador rotatório (Tecnal Brasil, modelo TE-211). A amostra resultante da concentração foi ainda liofilizada em um liofilizador Liotop (Liobras, modelo L101), para posterior armazenamento na forma sólida antes de ser analisada por cromatografia líquida de alta eficiência e avaliada sua capacidade antioxidante.

\subsection{Determinação de fenóis totais pelo método Folin-Ciocalteu}

A determinação de compostos fenólicos totais foi realizada em triplicata para cada extrato fenólico, empregando-se a metodologia descrita por Singleton e Rossi (1965), com as modificações de Palomino et al. (2009).

Com o objetivo de expressar os resultados como equivalentes de ácido gálico (mg de ácido gálico/g de resíduo), realizou-se uma curva de calibração com esse padrão nas concentrações de 50 a $500 \mu \mathrm{g} \mathrm{mL}^{-1}$. Para cada concentração, as análises foram realizadas em triplicata.

\subsection{Cromatografia líquida de alta eficiência}

A análise cromatográfica foi realizada em um cromatógrafo líquido de alta eficiência (analítico) modelo LC-10AD (Shimadzu), acoplado a um detector ultravioleta em arranjo de fotodiodos, modelo SPD-M10A (Shimadzu), com sistema de aquisição e tratamento de dados LCsolution (Shimadzu). Foi utilizada coluna tipo octadecil silano $\left(\mathrm{C}_{18}\right)$, modelo LUNA (Phenomenex ${ }^{\circledR}$ ) de $250 \mathrm{~mm} \times 4,6 \mathrm{~mm}$ d.i., com partículas de $5 \mu \mathrm{m}$ de tamanho médio.

O método para a identificação dos principais compostos fenólicos em resíduos de café baseou-se na metodologia exposta por Murthy e Naidu (2012) com modificações. O sistema de eluição consistiu em acetonitrila/água $\left(\mathrm{ACN} / \mathrm{H}_{2} \mathrm{O} 5: 95 \mathrm{v} / \mathrm{v}\right.$ ) (solvente $\mathrm{A}$ ) e $\mathrm{ACN} / \mathrm{H}_{2} \mathrm{O}(80: 20 \mathrm{v} / \mathrm{v})$ (solvente $\mathrm{B}$ ), sendo as duas fases acidificadas, respectivamente, com 0,035\% e 0,025\% de ácido trifluoroacético (TFA). Foi utilizada eluição por gradiente, incrementando-se o solvente $\mathrm{B}$ de 0 a $20 \%$ em 20 min e atingindo-se $50 \%$ de B em 50 min. A quantificação do ácido clorogênico foi realizada por meio do método de padrão externo. A detecção foi realizada em $\lambda=230 \mathrm{~nm}$ e $330 \mathrm{~nm}$.

\subsection{Determinação da capacidade antioxidante através do método de sequestro de radicais livres (DPPH')}

A avaliação da capacidade antioxidante foi realizada segundo o método de Brand-Williams et al. (1995) com algumas modificações. Alíquotas das soluções estoque dos extratos foram diluídas em etanol nas concentrações de 10 a $400 \mu \mathrm{g} \mathrm{mL}^{-1}$. O decaimento da absorbância das amostras depois de 30 minutos foi medida a $\lambda=515 \mathrm{~nm}$. Foram avaliadas também soluções etanólicas dos padrões rutina e quercetina, na faixa de concentração de 0,4 a $8,0 \mu \mathrm{g} \mathrm{mL} \mathrm{m}^{-1}$, para posterior comparação da capacidade antioxidante. A capacidade sequestradora do radical $\mathrm{DPPH}^{\bullet}$ foi calculada com a Equação 1:

$\%$ de DPPH'sequestrado $=\left(\frac{A_{\text {branco }}-A_{\text {amostra }}}{A_{\text {branco }}}\right) \times 100$

Em que $A_{\text {branco }}$ é a absorbância do controle e $A_{\text {amostra, }}$ a absorbância em presença do extrato fenólico.

Foi calculado o parâmetro $\mathrm{CE}_{50}$ (concentração efetiva média), que representa a concentração de amostra necessária para sequestrar $50 \%$ dos radicais de DPPH. $\mathrm{O} \mathrm{CE}_{50}$ foi obtido através do gráfico \% $\mathrm{DPPH}$ • reduzido versus concentração.

\subsection{Determinação da capacidade antioxidante avaliando-se a ação redutora sobre radicais peroxila}

A ação redutora dos extratos sobre radicais peroxila foi avaliada segundo o método de Pino et al. (2003) com modificações. Esse baseia-se no decaimento da fluorescência da piranina quando submetida à oxidação por radicais peroxila.

A partir das soluções estoque dos extratos foram preparadas diferentes diluições etanólicas com concentração de 1 a $40 \mu \mathrm{g} \mathrm{mL}-1$. Um volume de $150 \mu \mathrm{L}$ da solução do composto fluorescente piranina $(5 \mu \mathrm{M})$ foi incubado em placa preta de leitura de fluorescência, com $125 \mu \mathrm{L}$ da solução de AAPH $(40 \mu \mathrm{M})$ em PBS a $37^{\circ} \mathrm{C}$, na ausência ou presença de $25 \mu \mathrm{L}$ das soluções dos extratos. $O$ decaimento da fluorescência da piranina foi monitorado utilizando-se os comprimentos de onda de excitação de 485 nm e de emissão de 528 nm, em uma Leitora de Microplacas Multidetecção Synergy HT (BioTek). A capacidade antioxidante foi avaliada em função do tempo de atraso no decaimento da fluorescência da piranina, provocado pela presença dos extratos. A leitura foi realizada para cada concentração em triplicata e o 
controle da leitora foi feito com o software de análises de dados Gen5. O mesmo procedimento foi realizado com as soluções etanólicas do padrão ácido gálico, na faixa de concentração de 0,5 a $50 \mu \mathrm{g} \mathrm{mL} \mathrm{m}^{-1}$. Foram avaliados dois controles, o controle branco, contendo piranina, etanol e PBS, e o controle positivo, contendo piranina, etanol e AAPH.

A porcentagem do radical peroxila sequestrado foi calculada com a Equação 2:

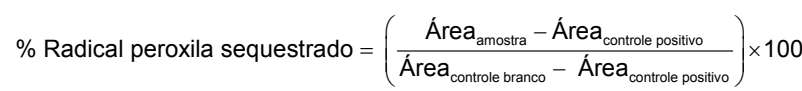

O $\mathrm{CE}_{50}$ foi obtido por meio do gráfico de \% Radical peroxila sequestrado versus concentração.

\subsection{Análise estatística}

As análises foram realizadas em triplicata. Os dados amostrais foram submetidos à análise de variância (ANOVA) e as médias foram comparadas pelo teste de Tukey por meio do programa Statistica 7.0 (StatSoft Inc., Oklahoma, USA). Os tratamentos foram considerados significativos para $P \leq 0,05$.

\section{Resultados e discussão}

\subsection{Compostos fenólicos nos extratos}

Os resíduos de café estudados apresentaram presença de compostos fenólicos totais entre 72,88 e 159,50 mg AG g ${ }^{-1}$ resíduo (Tabela 1). O resíduo com maior teor de compostos fenólicos totais foi a casca de café, apresentando também o maior conteúdo de ácido clorogênico.

A polpa de café apresentou menor teor de compostos fenólicos que a casca de café. Esse resultado é esperado se se levar em consideração que, no processamento por via seca, retira-se em conjunto, mediante o descasque, a casca, a polpa e o pergaminho, tudo em uma única etapa, e se dá o nome de casca ao resíduo gerado. No entanto, no processamento por via úmida, cada camada do grão verde de café é removida em diferentes etapas. Sendo assim, espera-se que o resíduo gerado no processamento seco seja mais rico em compostos fenólicos, pois contém todas as partes do fruto que envolvem a semente de café.

Não foi detectado ácido clorogênico na borra de café, portanto a presença de fenóis totais nesse resíduo pode ser atribuída a outros compostos, como cafeína e trigonelina, já reportados no estudo de Esquivel e Jiménez (2012). Segundo a literatura, na bebida de café, os polifenóis são quase que inteiramente ácido clorogênico, uma xícara da bebida (200 mL) pode conter cerca de 20 a 675 mg de ácido clorogênico (DE MARIA; MOREIRA, 2004; GARAMBONE; ROSA, 2007). Dessa forma, a ausência do ácido clorogênico na borra de café avaliada indica que o processamento do grão para a obtenção de café solúvel não retirou o ácido clorogênico do café processado, garantindo que ele esteja presente na bebida.

\subsection{Capacidade antioxidante através do método de sequestro de radicais livres (DPPH)}

Por meio das leituras de absorbância para cada amostra, os valores de \% DPPH • reduzidos foram calculados (Tabela 2) para obtenção dos valores de $\mathrm{CE}_{50}$. Vários testes foram realizados a fim de se avaliar o intervalo de concentração adequado para cada extrato atingir o valor de $\mathrm{CE}_{50}$. Para essa avaliação considerou-se que concentrações maiores que $100 \mu \mathrm{g} \mathrm{mL}^{-1}$ nos extratos indicam baixa capacidade antioxidante. Assim, o extrato da borra de café não atingiu o valor $\mathrm{CE}_{50}$ porque a maior concentração avaliada (400 $\mu \mathrm{g} \mathrm{mL} \mathrm{m}^{-1}$ ) foi considerada muito alta para atribuir-se a ela capacidade antioxidante.

A capacidade antioxidante foi avaliada por meio do gráfico de \% DPPH• reduzido em função da concentração dos extratos. Os valores dos $\mathrm{CE}_{50}$ dos extratos foram calculados usando-se a equação de primeira ordem obtida do gráfico de cada amostra.

Foi obtido o valor de $\mathrm{CE}_{50}=30,4 \pm 0,40$ para o extrato da casca de café e de 66,1 \pm 0,87 para o extrato da polpa de café. Quanto menor o valor de $\mathrm{CE}_{50}$, maior é o consumo do radical $\mathrm{DPPH}^{\bullet}$ pela amostra e, por conseguinte, maior é a capacidade antioxidante. Por sua parte, o extrato da borra de café apresentou $\mathrm{CE}_{50}>100$.

Tabela 1. Composição fenólica dos resíduos de café.

\begin{tabular}{|c|c|c|}
\hline Amostras & $\begin{array}{l}\text { Teor de fenóis totais } \\
\text { (mg AG g-1 resíduo) }\end{array}$ & $\begin{array}{l}\text { Conteúdo de ácido } \\
\text { clorogênico } \\
\text { ( } \mu \mathrm{g} \mathrm{mL}^{-1} \pm \text { d.p.) }\end{array}$ \\
\hline $\mathrm{CA}$ & $159,50 \pm 1,06^{A}$ & $1927,63 \pm 0,73^{A}$ \\
\hline PA & $72,88 \pm 0,71^{c}$ & $516,83 \pm 1,50^{\mathrm{B}}$ \\
\hline BA & $77,25 \pm 0,24^{\mathrm{B}}$ & - \\
\hline
\end{tabular}

CA: extrato acetônico da casca; PA: extrato acetônico da polpa; BA: extrato acetônico da borra. As médias que não compartilham uma letra na mesma coluna são significativamente diferentes $(P \leq 0,05)$.

Tabela 2. Porcentagem de DPPH ${ }^{\bullet}$ reduzido pelas amostras e padrões.

\begin{tabular}{ccc} 
Amostras & $\begin{array}{c}\text { Concentração } \\
\left(\boldsymbol{\mu g} \mathbf{~ m L}^{-1}\right)\end{array}$ & $\%$ DPPH' reduzido \\
\hline CA & $10,0-60,0$ & $23,1-78,0$ \\
PA & $20,0-100,0$ & $25,8-67,6$ \\
BA & $40,0-400,0$ & $15,1-28,6$ \\
Quercetina & $0,4-8,0$ & $6,5-85,3$ \\
(padrão) & $0,4-8,0$ & $6,0-62,8$ \\
Rutina (padrão) & \\
\hline
\end{tabular}

CA: extrato acetônico da casca; PA: extrato acetônico da polpa; BA: extrato acetônico da borra. 
Verificou-se que a capacidade antioxidante dos extratos e padrões segue a seguinte ordem: quercetina $>$ rutina $>$ casca de café $(C A)>$ polpa de café $(P A)>$ borra de café (Figura 1).

\subsection{Determinação da capacidade redutora de radicais peroxila}

Neste ensaio avaliou-se a reatividade das moléculas antioxidantes presentes nos extratos com os radicais peroxila derivados do AAPH. Foi calculada a porcentagem de radical peroxila sequestrado (Tabela 3 ) e, posteriormente, obtidos os valores de $\mathrm{CE}_{50}$.

A capacidade antioxidante foi avaliada por meio do gráfico de \% Radical peroxila sequestrado em função da concentração dos extratos. Os valores de $\mathrm{CE}_{50}$ dos extratos foram calculados usando-se a equação de primeira ordem obtida do gráfico de cada amostra.

Segundo a Figura 2, a capacidade antioxidante dos extratos e o padrão seguem a seguinte ordem: casca de café $(C A)>$ polpa de café $(P A)>$ ácido gálico > borra de café.

Deve-se ressaltar que os extratos da casca e da polpa de café apresentaram melhor capacidade antioxidante que o padrão ácido gálico, evidenciando sua efetividade no sequestro de radicais peroxila. Com relação à borra de café, observou-se que não houve incremento significativo da atividade antioxidante quando foram avaliadas diferentes concentrações do extrato, e em consequência, não foi atingido o valor de $\mathrm{CE}_{50}$.

Os resultados da ação redutora sobre radicais peroxila concordam com os obtidos para o radical DPPH: em ambos os casos o extrato com maior capacidade antioxidante foi o da casca de café.

A capacidade antioxidante evidenciada pelos extratos in natura pode se relacionar com a elevada concentração de ácido clorogênico presente. O ácido clorogênico faz parte do grupo de ácidos fenólicos que se caracterizam por ter um anel benzênico, um grupo carboxílico e um ou mais grupos de hidroxila e/ou metoxila na molécula, o que Ihes confere propriedades antioxidantes, tanto para os alimentos como para o organismo (SOARES, 2002). A ação antioxidante do ácido clorogênico atribui-se à presença de um grupo orto-di-hidroxila no anel aromático do ácido cafeico, que atuaria como um receptor de radicais livres (LUZIA et al., 1998).

A capacidade antioxidante do ácido clorogênico tem sido demonstrada em diferentes estudos. Por exemplo, isômeros do ácido clorogênico isolados da ameixa seca demonstraram ação antioxidante quando avaliados em ensaios de eliminação de radicais superóxido e inibição contra oxidação do metil linoleato (NAKATANI et al., 2000). O ácido clorogênico, encontrado em extratos de cenoura, bardana, damasco e ameixa-preta, mostrou ser um potente inibidor da peroxidação lipídica in vitro e evidenciou uma possível ação anticarcinogênica também in vivo (KASAl et al., 2000). Isômeros derivados do ácido clorogênico isolados das folhas de bambu apresentaram alta capacidade de eliminar radicais livres, radicais superóxido, além de inibirem a peroxidação lipídica (KWEON et al., 2001).

Esses resultados sugerem a possibilidade de reutilização dos resíduos da indústria cafeeira para a obtenção de compostos antioxidantes, pois tais compostos têm inúmeras aplicações nas áreas de alimentos, cosméticos e na indústria farmacêutica (AO et al., 2011; BALLESTEROS et al., 2014).

Tabela 3. Porcentagem de radical peroxila sequestrado pelas amostras e padrão.

\begin{tabular}{ccc} 
Amostras & $\begin{array}{c}\text { Concentração } \\
\left(\boldsymbol{\mu g} \mathbf{~ m L}^{-1}\right)\end{array}$ & $\begin{array}{c}\text { \% Radical } \\
\text { peroxila } \\
\text { sequestrado }\end{array}$ \\
\hline CA & $1,0-10,0$ & $14,5-70,5$ \\
PA & $1,0-10,0$ & $9,7-57,3$ \\
BA & $5,0-40,0$ & $7,9-13,5$ \\
Ácido gálico & $5,0-50,0$ & $8,3-109,3$ \\
(padrão) & \\
\hline
\end{tabular}

CA: extrato acetônico da casca; PA: extrato acetônico da polpa; BA: extrato acetônico da borra.

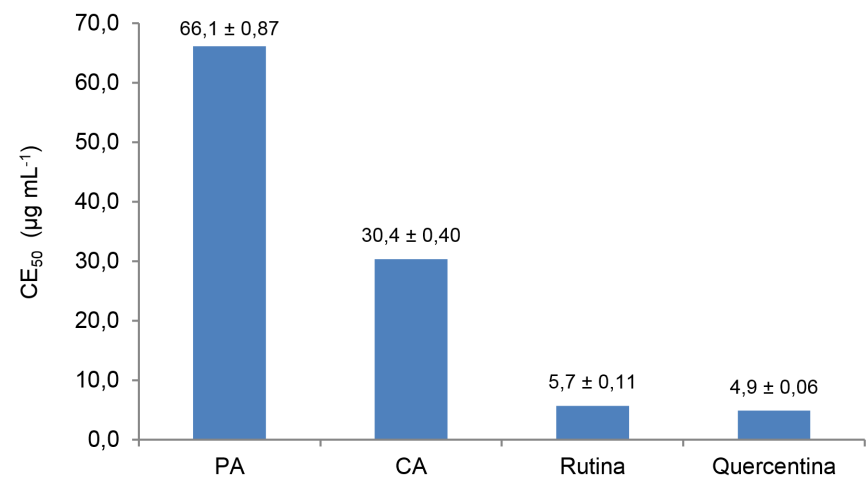

Figura 1. Representação gráfica do $\mathrm{CE}_{50}$ obtido pelo método do DPPH para os extratos e os padrões.

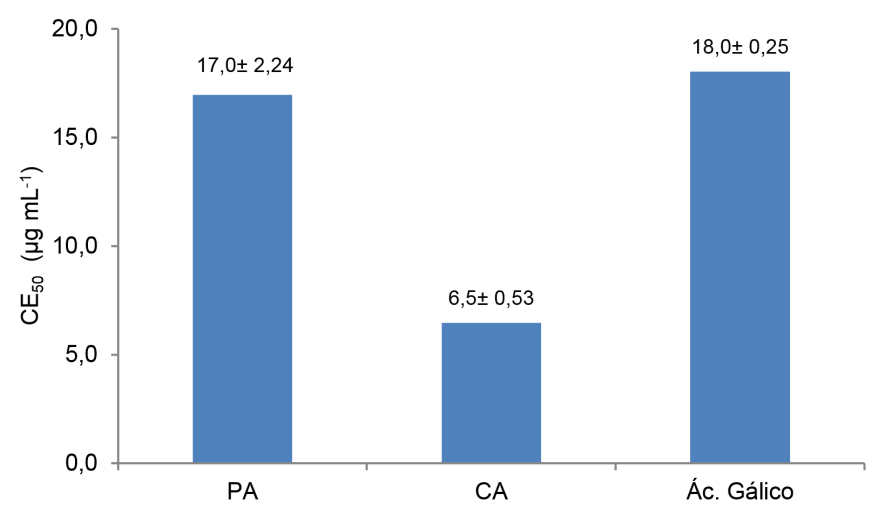

Figura 2. Representação gráfica do $C_{50}$ obtido na avaliação da ação redutora dos extratos e o padrão. 


\section{Conclusão}

Os resíduos de café, tais como a casca, a polpa e a borra, contêm quantidades apreciáveis de compostos fenólicos. Dentre os resíduos avaliados, o maior teor de fenóis foi encontrado na casca de café.

Os extratos acetônicos da casca e da polpa de café apresentaram capacidade antioxidante, a qual foi superior à apresentada pelo ácido gálico quando avaliada a ação redutora sobre radicais peroxila.

A capacidade antioxidante dos extratos pode ser correlacionada com o teor de compostos fenólicos, determinado pelo método de Folin-Ciocalteu, e com a presença de ácido clorogênico, quantificado por CLAE. Evidentemente, os compostos fenólicos presentes nesses resíduos conferem aos extratos atividade sequestradora e poder redutor de radicais livres.

A recuperação de ácido clorogênico pode ser uma alternativa para a indústria farmacêutica e de alimentos, levando em consideração a reconhecida atividade biológica desse composto fenólico. Os resíduos da indústria cafeeira têm potencial para serem utilizados em bioprocessos, avaliando a extração e/ou a produção de compostos com valor biotecnológico.

\section{Agradecimentos}

Os autores agradecem ao Núcleo de Pesquisa NuBBE do Instituto de Química de Araraquara, Departamento de Química Orgânica, Universidade Estadual Paulista (Brasil), pela permissão de realizar as análises de CLAE e de atividade antioxidante em seus laboratórios.

\section{Referências}

AJILA, C. M.; BRAR, S. K.; VERMA, M.; TYAGI, R. D.; VALÉRO, J. R. Solid-state fermentation of apple pomace using Phanerocheate chrysosporium - I iberation and extraction of phenolic antioxidants. Food Chemistry, London, v. 126, n. 3, p. 1071-1080, 2011. http://dx.doi.org/10.1016/j. foodchem.2010.11.129.

AO, C.; HIGA, T.; KHANH, T. D.; UPADHYAY, A.; TAWATA, S. Antioxidant phenolic compounds from Smilax sebeana Miq. LWT - Food Science and Technology, Waltham, v. 44, n. 7, p. 1681-1686, 2011.

BALLESTEROS, L. F.; TEIXEIRA, J. A.; MUSSATTO, S. I. Chemical, functional, and structural properties of spent coffee grounds and coffee silverskin. Food and Bioprocess Technology, New York, v. 7, n. 12, p. 3493-3503, 2014. http://dx.doi.org/10.1007/ s11947-014-1349-z.

BRAND-WILLIAMS, W.; CUVELIER, M. E.; BERSET, C. Use of a free radical method to evaluate antioxidant activity. LWT - Food Science and Technology, Waltham, v. 28, n. 1, p. 25-30, 1995.
CLIFFORD, M. N.; RAMÍREZ-MARTINEZ, J. R. Phenols and caffeine in wet-processed coffee beans and coffee pulp. Food Chemistry, London, v. 40, n. 1, p. 35-42, 1991. http://dx.doi. org/10.1016/0308-8146(91)90017-I.

DE MARIA, A. C. B.; MOREIRA, R. F. A. Métodos para análise de ácido clorogênico. Química Nova, São Paulo, v. 27, n. 4, p. 586-592, 2004.

ESQUIVEL, P.; JIMÉNEZ, V. M. Functional properties of coffee and coffee by-products. Food Research International, Barking, v. 46, n. 2, p. 488-495, 2012. http://dx.doi.org/10.1016/j. foodres.2011.05.028.

FAN, L.; PANDEY, A.; MOHAN, R.; SOCCOL, C. R. Use of various coffee industry residues for the cultivation of Pleurotus ostreatus in solid state fermentation. Acta Biotechnology, Weinheim, v. 20, n. 1, p. 41-52, 2000. http://dx. doi.org/10.1002/ abio.370200108.

GARAMBONE, E.; ROSA, G. Possíveis benefícios do ácido clorogênico à saúde. Alimentos e Nutrição, Marilia, v. 18, n. 2, p. 229-235, 2007.

KASAI, H.; FUKADA, S.; YAMAIZUMI, Z.; SUGIE, S.; MORI, $H$. Action of chlorogenic acid in vegetables and fruits as an inhibitor of 8-hydroxydeoxyguanosine formation in vitro and in a rat carcinogenesis model. Food and Chemical Toxicology, Oxford, v. 38, n. 5, p. 467-471, 2000. http://dx.doi.org/10.1016/ S0278-6915(00)00014-4. PMid:10762733.

KWEON, M. H.; HWANG, H. J.; SUNG, H. C. Identification and antioxidant activity of novel chlorogenic acid derivatives from bamboo (Phyllostachys edulis). Journal of Agricultural and Food Chemistry, Easton, v. 49, n. 10, p. 4646-4655, 2001. http:// dx.doi.org/10.1021/jf010514x. PMid:11600002.

LUZIA, M. R.; TRUGO, L. C.; DA PAIXÃO, K. C. C.; MARCÍLIO, R.; DE MARIA, C. A. B.; QUINTEIRO, L. M. C. Effect of 5-caffeoylquinic acid in the presence of metal chelators on soybean oil oxidative stability. Lebensmittel-Wissenschaft \& Technologie, London, v. 31, n. 1, p. 64-68, 1998. http://dx.doi. org/10.1006/fstl.1997.0294.

MURTHY, P. S.; NAIDU, M. M. Recovery of phenolic antioxidants and functional compounds from coffee industry by-products. Food and Bioprocess Technology, New York, v. 5, n. 3, p. 897-903, 2012. http://dx. doi.org/10.1007/s11947-010-0363-z.

MUSSATTO, S. I.; MACHADO, E. M. S.; MARTINS, S.; TEIXEIRA, J. A. Production, composition, and application of coffee and its industrial residues. Food and Bioprocess Technology, New York, v. 4, n. 5, p. 661-672, 2011. http://dx.doi.org/10.1007/ s11947-011-0565-z.

NAKATANI, N.; KAYANO, S.; KIKUZAKI, H.; SUMINO, K.; KATAGIRI, K.; MITANI, T. Identification, quantitative determination, and antioxidative activities of chlorogenic acid isomers in prune (Prunus domestica L.). Journal of Agricultural 
and Food Chemistry, Easton, v. 48, n. 11, p. 5512-5516, 2000. http://dx.doi.org/10.1021/jf000422s. PMid:11087511.

PALOMINO, L. R.; DEL BIANCHI, V. L. Efeito da fermentação fúngica no teor de compostos fenólicos em casca de café robusta. Semina: Ciências Agrárias, Londrina, v. 36, n. 2, p. 777-786, 2015.

PALOMINO, L. R.; GARCÍA, C. M.; GIL, J. H.; ROJANO, B. A.; DURANGO, D. L. Determinación del contenido de fenoles y evaluación de la actividad antioxidante de propóleos recolectados en el departamento de Antioquia (Colombia). VITAE, Medellín, v. 16, n. 3, p. 388-395, 2009.

PANDEY, A.; SOCCOL, C.; NIGAM, P.; BRAND, D.; MOHAN, R.; ROUSSOS, S. Biotechnological potential of coffee pulp and coffee husk for bioprocesses. Biochemical Engineering Journal, Amsterdam, v. 6, n. 2, p. 153-162, 2000. http://dx. doi. org/10.1016/S1369-703X(00)00084-X. PMid:10959086.

PINO, E.; CAMPOS, A. M.; LISSI, E. 8-Hydroxy-1,3,6-pyrene trisulfonic acid (pyranine) bleaching by 2,2'-azobis(2amidinopropane) derived peroxyl radicals. International Journal of Chemical Kinetics, New York, v. 35, n. 10, p. 525-531, 2003. http://dx.doi.org/10.1002/kin. 10151.
PRATA, E. R. B. A.; OLIVEIRA, L. S. Fresh coffee husks as potential sources of anthocyanins. LWT - Food Science and Technology, Waltham, v. 40, n. 9, p. 1555-1560, 2007.

RAMALAKSHMI, K.; RAO, L. J. M.; TAKANO-ISHIKAWA, Y.; GOTO, M. Bioactivities of low-grade green coffee and spent coffee in different in vitro model systems. Food Chemistry, London, v. 115, n. 1, p. 79-85, 2009. http://dx.doi.org/10.1016/j. foodchem.2008.11.063.

RAMIREZ-CORONEL, M. A.; MARNET, N.; KUMAR-KOLLI, V. S.; ROUSSOS, S.; GUYOT, S.; AUGUR, C. Characterization and estimation of proanthocyanidins and other phenolics in coffee pulp (Coffea arabica) by thiolysis-high-performance liquid chromatography. Journal of Agricultural and Food Chemistry, Easton, v. 52, n. 5, p. 1344-1349, 2004. http://dx.doi.org/10.1021/ jf035208t. PMid:14995144.

SINGLETON, V. L.; ROSSI, J. A. Colorimetry of total phenolics with phosphomolybdic-phosphotungstic acid reagents. American Journal of Enology and Viticulture, Davis, v. 16, n. 3, p. 144-158, 1965.

SOARES, S. E. Ácidos fenólicos como antioxidantes. Revista de Nutrição, Campinas, v. 15, n. 1, p. 71-81, 2002. 